\title{
Dimensiones transversales del arco maxilar y esqueléticas en pacientes con secuela de fisura labio alveolo palatina unilateral.
}

Aliaga-Del Castillo A ${ }^{1}$, Reyna-Gutiérrez $\mathrm{J}^{1}$, Soldevilla-Galarza L ${ }^{2}$, Mattos-Vela $\mathrm{M}^{3}$, Aliaga-Del Castillo $\mathrm{R}^{4}$. Dimensiones transversales del arco maxilar y esqueléticas en pacientes con secuela de fisura labio alveolo palatina unilateral. Rev Estomatol Herediana. 2012; 22(1):20-25.

\section{RESUMEN}

Objetivos: El propósito de este estudio fue evaluar las dimensiones transversales del arco maxilar (ancho intercanino y ancho intermolar) y esqueléticas (ancho maxilar y ancho facial) en pacientes con secuela de Fisura Labio Alveolo Palatina Unilateral (FLAPU) y compararlas con las de pacientes no fisurados. Material y Métodos: Se utilizaron modelos de estudio y radiografías postero-anteriores de 94 niños de 6 a 15 años de edad atendidos en el Instituto Especializado en Salud del Niño, 47 con secuela de FLAPU y 47 no fisurados. Se tomaron fotografías digitales para la evaluación de los modelos de estudio, las distancias se midieron en el maxilar superior para el ancho intercanino de centroide a centroide de caninos deciduos o permanentes contralaterales y para el ancho intermolar de centroide a centroide de primeras molares permanentes contralaterales. Se realizaron trazos cefalométricos utilizando los criterios de Ricketts para la evaluación de las radiografías. Resultados: En los pacientes con secuela de FLAPU la distancia promedio \pm desviación estándar para el ancho intercanino, ancho intermolar, ancho maxilar, y ancho facial, fue de $26,94 \pm 4,30 \mathrm{~mm}, 47,77 \pm 4,61 \mathrm{~mm}, 65,06 \pm 4,96 \mathrm{~mm}$ y $125,50 \pm 5,42$ $\mathrm{mm}$, respectivamente; en los pacientes no fisurados fue de $31,77 \pm 2,03 \mathrm{~mm}, 47,86 \pm 2,84 \mathrm{~mm}, 65,04$ $\pm 3,47 \mathrm{~mm}$ y $124,38 \pm 6,60 \mathrm{~mm}$, respectivamente Conclusiones: En las dimensiones transversales del arco maxilar, el ancho intercanino fue menor en los pacientes con secuela de FLAPU. Sin embargo, no existieron diferencias entre las dimensiones transversales esqueléticas en los dos grupos de pacientes.

PALABRAS CLAVE: Labio hendido, paladar hendido, arco dental/anomalías, maxilar/anomalías.

Transversal arch maxillary and skeletal dimensions in patients with unilateral cleft lip alveolus and palate sequel.

\begin{abstract}
Objectives: The purpose of this study was to evaluate transverse arch maxillary dimensions (intercanine width and intermolar width) and transverse skeletal dimensions (maxillary width and facial width) in patients with sequel of Unilateral Cleft Lip Alveolus and Palate (UCLAP) and compared with those of patients without cleft. Material and methods: Was used dental casts and posteroanterior radiographs of 94 children aged 6 to 15 belonging at Specialized Institute of Children's Health, 47 belonging to the group with UCLAP sequel and 47 belonging to noncleft patients. Digital photographs were taken for the assessment of dental casts, the distances were measured in the maxilla for the intercanine width centroid to centroid of deciduous or permanent contralateral canines and for the intermolar width centroid to centroid of first permanent contralateral molars. Cephalometric traces were performed following Ricketts criteria for radiographs evaluation. Results: In patients with UCLAP sequel the distance average \pm standard deviation for intercanine width, intermolar width, maxillary width and facial width was $26.94 \pm 4.30 \mathrm{~mm}, 47.77 \pm 4.61 \mathrm{~mm}, 65,06 \pm 4.96 \mathrm{~mm}$ and $125.50 \pm 5.42 \mathrm{~mm}$, respectively; In noncleft patients the average distance for intercanine width, intermolar width, maxillary width and facial width was $31.77 \pm 2.03 \mathrm{~mm}, 47.86 \pm 2.84 \mathrm{~mm}, 65.04 \pm 3.47 \mathrm{~mm}$ and $124.38 \pm 6.60 \mathrm{~mm}$, respectively. Conclusions: In the transverse dimensions of the maxillary arch, intercanine width was lower in patients with sequel of UCLAP. However, there were no differences between the skeletal transverse dimensions in the two groups of patients.
\end{abstract}

KEY WORDS: Cleft lip, cleft palate, dental arch/abnormalities, maxilla/abnormalities.

\section{Arón Aliaga Del Castillo' Julio Reyna Gutiérrez ${ }^{1}$ Luciano Soldevilla Galarza ${ }^{2}$ Manuel Mattos Vela ${ }^{3}$ Rosalinda Aliaga Del Castillo ${ }^{4}$}

\section{'Cirujano Dentista.}

Egresado Facultad de Odontología Universidad Nacional Mayor de San Marcos. Lima, Perú.

\section{${ }^{2}$ Cirujano Dentista.}

Docente del Departamento de Estomatología Pediátrica, Facultad de Odontología Universidad Nacional Mayor de San Marcos. Lima, Perú.

\section{${ }^{3}$ Cirujano Dentista.}

Docente del Departamento de Estomatología Biosocial, Facultad de Odontología Universidad Nacional Mayor de San Marcos. Lima, Perú.

\section{${ }^{4}$ Cirujano Dentista}

Especialista en Estomatología Pediátrica, Universidad Peruana Cayetano Heredia. Lima, Perú

\section{Correspondencia}

Arón Aliaga-Del Castillo

Jr. J.J. Pasos 490 Int. 401. Pueblo Libre. Lima 21, Perú

Telefono: 511 - 3324318 / 511996845677

E-mail: a_aliaga@hotmail.com

\section{Introducción}

La fisura labial con o sin fisura palatina ocurre en uno de cada mil nacimientos (1), y el lado izquierdo es el más afectado (1-3).

Algunas de las características más comunes en estos pacientes son: patrón de crecimiento deficiente en el tercio medio de la cara $(4,5)$, alteración en la forma de arco, asimetría causada por una in- hibición en el desarrollo maxilar en el sentido transversal y sagital por el tejido cicatricial presente en el paladar y en el labio de los pacientes que han sido sometidos a cirugía (5-7) o por la ausencia de tejido blando y óseo en los pacientes que nunca han sido intervenidos quirúrgicamente $(8,9)$.

Los pacientes con labio y paladar fisurado unilateral tienen una deficiencia en la forma y tamaño de los tejidos afectados y esto causa una asimetría en el ámbito facial, esquelético y dental; además, presentan un severo colapso transversal de los arcos maxilares, pérdida de dientes y otras irregularidades causadas por interferencias estructurales y funcionales. Este colapso de arco es mayor en la zona de los caninos y disminuye progresivamente ha- 
cia la región molar; es causado por una rotación medial del segmento del lado afectado $(4,9,10)$ y la falta de continuidad en el reborde alveolar y por la ausencia de la sutura media palatina $(11,12)$.

Por lo dicho anteriormente suele suponerse que las dimensiones transversales del arco maxilar y esqueléticas son menores en los pacientes fisurados, pero no tenemos sustento científico para afirmar que ese fenómeno ocurre en una población peruana ya que debido a la poca relevancia que el odontólogo general brinda a este tema, no se han realizado estudios al respecto en Perú.

La presente investigación tuvo como objetivo evaluar las dimensiones transversales del arco maxilar (ancho intercanino y ancho intermolar) y esqueléticas (ancho maxilar y ancho facial) en pacientes con secuela de Fisura Labio-Alveolo-Palatina Unilateral (FLAPU) y compararlas con las de pacientes no fisurados (NF).

\section{Material y Métodos}

El presente estudio es descriptivo, comparativo, retrospectivo y transversal.

La muestra se obtuvo por conveniencia, estuvo constituida por 94 modelos de estudio (47 FLAPU y 47 NF) y por 94 radiografías posteroanteriores (47 FLAPU y 47 NF) de niños entre 6 a 15 años de edad (divididos en tres grupos: de 6 a 9, de 10 a 12 , y de 13 a 15 años de edad) atendidos en el Servicio de Ortodoncia y Ortopedia Maxilar del Instituto Especializado en Salud del Niño (IESN), entre enero del 2000 y Octubre del 2009. Los pacientes con secuela de FLAPU pertenecían a la Unidad de Fisurados del Servicio ya mencionado y los pacientes NF (grupo control) fueron pacientes que presentaban maloclusión de Angle clase I y pertenecían al mismo Servicio. Los criterios de exclusión fueron: marcada inclinación de caninos, lesiones cariosas interproximales en segundas molares deciduas o segundas premolares, pérdida de segundas molares deciduas y tratamiento ortodóntico previo para ambos grupos de pacientes; así como, síndrome asociado para el grupo con secuela de FLAPU y displasias transversales para el grupo NF. Los permisos se gestionaron en la Oficina de Docencia del IESN.

\section{Evaluación de modelos de estudio}

Se utilizaron modelos de estudio existentes en el archivo del Servicio de Ortodoncia y Ortopedia Maxilar. A partir de la digitalización de los modelos de estudio por medio de fotografías se midió el ancho intercanino (distancia entre los centroides de los caninos deciduos o permanentes superiores contralaterales) y el ancho intermolar (distancia entre los centroides de las primeras molares superiores permanentes contralaterales) (13).

Montaje y orientación de los modelos

Se realizó el procedimiento teniendo como referencia los procedimientos y técnicas utilizadas por Moyers et al. (13). Para encontrar el plano oclusal funcional se utilizó un paralelígrafo. Se orientó el plano oclusal colocando las cúspides mesiovestibulares de ambas primeras molares superiores permanentes y la cúspide vestibular de la segunda premolar superior izquierda o segunda molar decidua superior izquierda a una misma distancia vertical de la base del paralelígra-

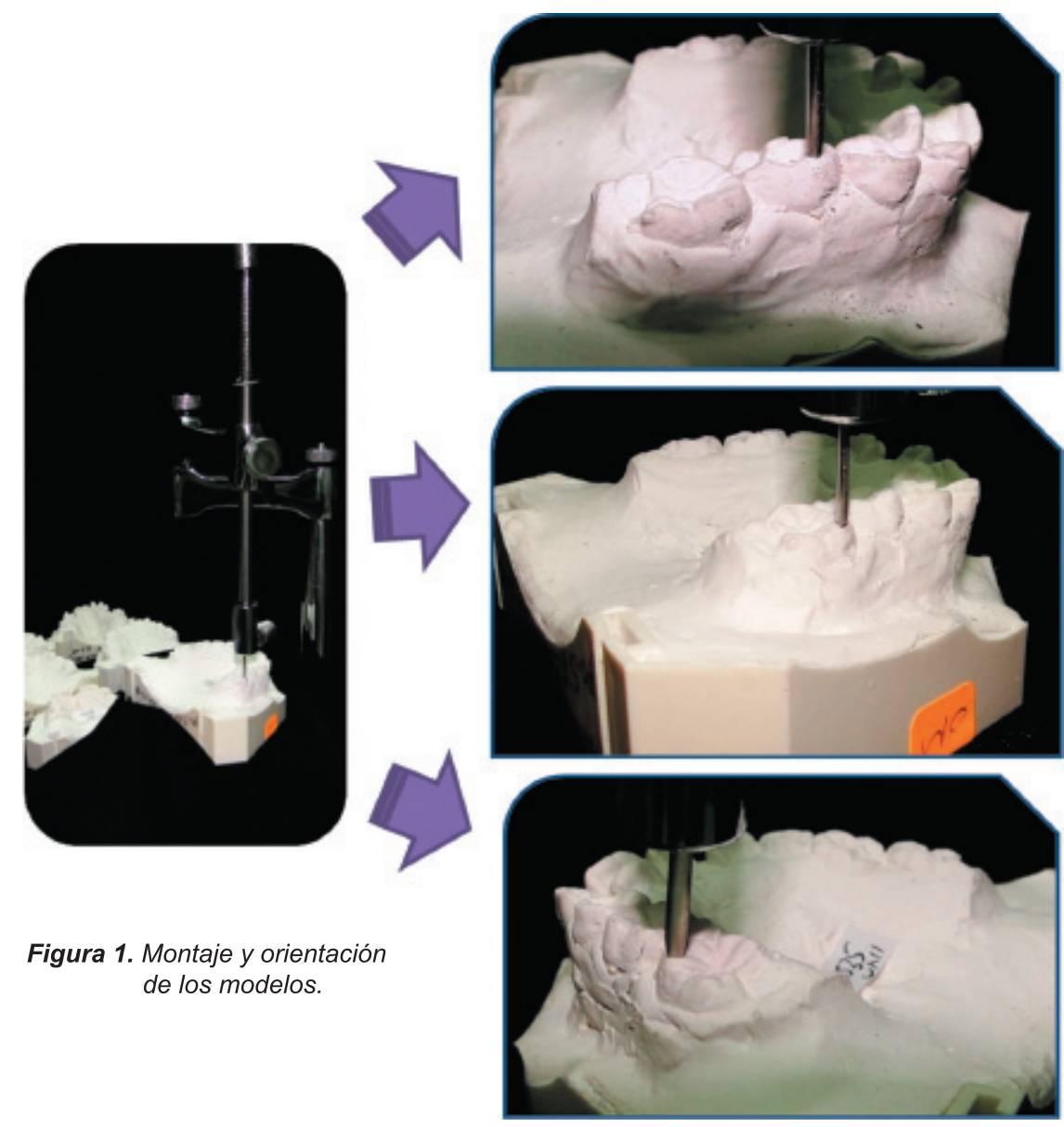


fo, para ello se buscó coincidencia entre los puntos mencionados y la punta seca metálica del estilete del paralelígrafo (Figura 1).

\section{Toma de fotografía digital}

La cámara digital se posicionó, con la ayuda de un trípode, con el lente en dirección ortoradial al objeto, en este caso el modelo de estudio, posicionado correctamente sobre el portamodelos del paralelígrafo. Para dar la escala a la fotografía se colocaron dos reglas milimetradas en forma de L sobre la superficie del portamodelos.

\section{Determinación del centroide}

Moyers et al. definen el centroide como el punto medio entre los dos puntos calculados de los puntos medios proximales y de los puntos ves tibular y palatino (13) (Figura 2).

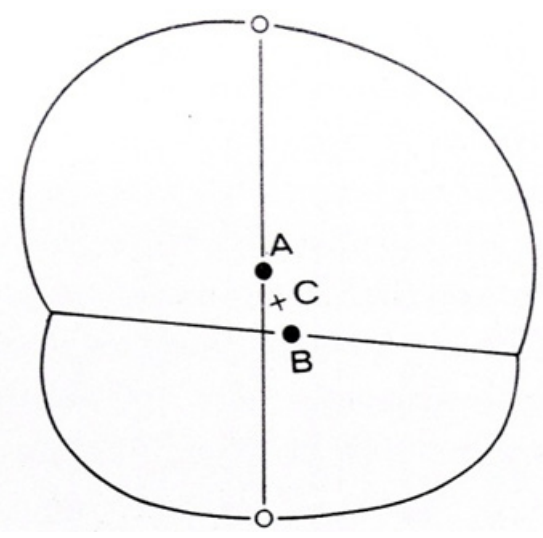

Figura 2. Determinación de los puntos medios y el centroide de una pieza dentaria. A: punto medio entre los puntos medios proximales mesial y distal. B: punto medio entre los puntos vestibular y palatino. C: el centroide, punto medio entre $A$ y $B$.

En el presente estudio, estos puntos se ubicaron para las molares: puntos proximales a nivel del surco central y puntos laterales a nivel del surco palatino y a nivel del surco vestibular; y para los caninos: puntos proximales a nivel del mayor

diámetro mesiodistal y los laterales a nivel de la máxima convexidad vestibular y palatina.

Evaluación de las radiografías posteroanteriores.

Se utilizaron radiografías poste

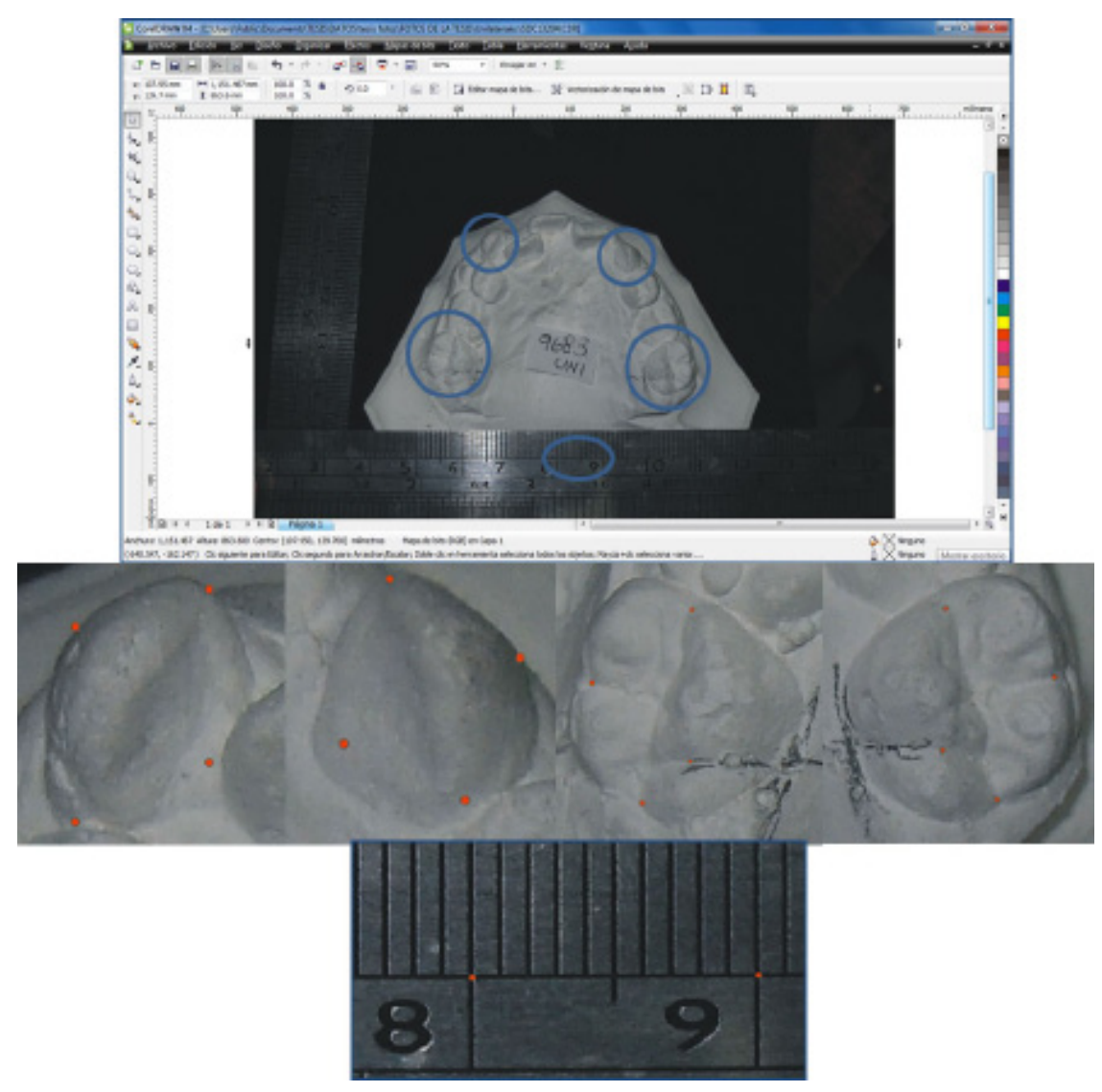

Figura 3. Análisis informático de las fotografías de los modelos de estudio.

Las fotografías se importaron al programa CorelDRAW X4. Este programa tiene incorporado un sistema de coordenadas que fue utilizado con dos fines: establecer las coordenadas de los puntos mesial, distal, palatino y vestibular de cada pieza dentaria en el formato $(\mathrm{X} ; \mathrm{Y})$; y establecer las coordenadas de los puntos que demarcaban $10 \mathrm{~mm}$ en la foto (L1 y L2), también en formato $(\mathrm{X} ; \mathrm{Y})$. Estos datos fueron transportados a una matriz de análisis específicamente desarrollada para este estudio en el programa informático Microsoft Excel 2007 la cual permitió obtener las coordenadas del centroide de cada pieza dentaria y calcular la distancia entre ellos (Figura 3). roanteriores existentes en el archivo del Servicio de Ortodoncia y Ortopedia Maxilar Los trazados cefalométricos se realizaron siguiendo los criterios establecidos por Ricketts (14). El ancho maxilar estuvo determinado por la distancia entre los puntos JL y JR (puntos bilaterales ubicados en la intersección de los contornos de la tuberosidad y pilar zigomático) y el ancho facial por la distancia entre los puntos ZA y AZ (puntos ubicados en el centro de la raíz del arco zigomático), así como lo describen también Gregoret (15) y Romani (16).

Los procedimientos arriba mencionados fueron realizados por un solo investigador de esa forma se trató 
de disminuir el sesgo en las mediciones.

\section{Análisis estadístico}

Para el análisis de los datos se utilizó el programa SPSS 15.0. Se aplicó la prueba de Kolmogorov Smirnov para evaluar la normalidad de la muestra y la prueba de Levene para evaluar la homogeneidad de varianzas de la muestra. Se usó la prueba $t$ de Student para comparar las medias de ambos grupos. Todas las pruebas se realizaron con un nivel de significancia del 0,05 .

\section{Resultados}

Se encontró que el ancho intercanino es menor en pacientes con secuela de FLAPU que en pacientes NF pero sólo en los grupos de 6 a 9 y de 10 a 12 años $(p<0,001)$. No se encontraron diferencias con respecto al sexo para cada grupo en particular $(\mathrm{p}>0,05)$ (Tabla 1). Para el ancho intermolar no existen diferencias entre ambos grupos, en ninguno de los grupos de edad y tampoco inter e intra sexos $(\mathrm{p}>0,05)$ (Tabla 2).

Para el ancho maxilar no existen diferencias entre ambos grupos de pacientes en ninguno de los tres grupos de edad, y tampoco inter e intra sexos $(p>0,05)$ (Tabla 3). Para el ancho facial no existen diferencias entre ambos grupos de pacientes, en ninguno de los tres grupos de edad, ni intra sexo $(\mathrm{p}>0.05)$; sin embargo, en el grupo de pacientes $\mathrm{NF}$, el ancho facial es mayor en el sexo masculino $(p=0,040)$ (Tabla 4).

\section{Discusión}

Motohashi et al (8), comprobaron que los pacientes fisurados mostraron una marcada deformidad facial en comparación con los pacientes no fisurados, caracterizada
Tabla 1. Comparación de ancho intercanino entre pacientes con secuela de FLAPU y pacientes no fisurados de 6 a 15 años atendidos en el IESN 2000-2009.

\begin{tabular}{lcccccccc}
\hline Covariables & \multicolumn{3}{c}{ Ancho intercanino(mm) } & \multicolumn{5}{c}{ Ancho intercanino (mm) } \\
& $\mathbf{n}$ & Media & $\mathbf{D E *}$ & $\mathbf{P}$ & $\mathbf{N}$ & Media & $\mathbf{D E}$ & $\mathbf{p}$ \\
\hline & 47 & 26,94 & 4,30 & & 47 & 31,77 & 2,03 & \\
Sexo & & & & $0,579 \dagger$ & & & & $0,496 \dagger$ \\
Masculino & 24 & 27,29 & 4,04 & & 20 & 32,01 & 1,71 & $0,000 \ddagger$ \\
Femenino & 23 & 26,58 & 4,61 & & 27 & 31,60 & 2,25 & $0,000 \ddagger$ \\
Edad (años) & & & & & & & & \\
6 a 9 & 21 & 27,93 & 3,47 & & 30 & 31,77 & 2,12 & 0,000 \\
10 a 12 & 16 & 25,00 & 4,82 & & 14 & 31,98 & 1,77 & 0,000 \\
13 a 15 & 10 & 27,96 & 4,33 & & 3 & 30,84 & 2,80 & 0,308 \\
\hline
\end{tabular}

* $\mathrm{DE}=$ Desviación estándar

$\uparrow$ Comparación inter sexos (entre masculino y femenino del mismo grupo).

† Comparación intra sexos (entre masculino y masculino, femenino y femenino de ambos grupos).

Tabla 2. Comparación de ancho intermolar entre pacientes con secuela de FLAPU y pacientes no fisurados de 6 a 15 años atendidos en el IESN 2000-2009.

\begin{tabular}{lcccccccc}
\hline & \multicolumn{3}{c}{ FLAPU } & \multicolumn{5}{c}{ NF } \\
Covariables & Ancho intermolar (mm) & \multicolumn{5}{c}{ Ancho intermolar (mm) } \\
& $\mathbf{n}$ & Media & $\mathbf{D E}^{*}$ & $\mathbf{P}$ & $\mathbf{N}$ & Media & $\mathbf{D E}$ & $\mathbf{p}$ \\
\hline & 47 & 47,77 & 4,61 & & 47 & 47,86 & 2,84 & \\
Sexo & & & & $0,937 \dagger$ & & & & $0,055 \dagger$ \\
Masculino & 24 & 47,83 & 4,90 & & 20 & 48,79 & 2,72 & $0,415 \ddagger$ \\
Femenino & 23 & 47,72 & 4,40 & & 27 & 47,18 & 2,79 & $0,539 \ddagger$ \\
Edad (años) & & & & & & & & \\
6 a 9 & 21 & 47,06 & 4,92 & & 30 & 47,42 & 2,80 & 0,765 \\
10 a 12 & 16 & 47,89 & 3,78 & & 14 & 48,46 & 2,79 & 0,647 \\
13 a 15 & 10 & 49,07 & 5,29 & & 3 & 49,52 & 3,47 & 0,894 \\
\hline
\end{tabular}

* DE= Desviación estándar

$\uparrow$ Comparación inter sexos (entre masculino y femenino del mismo grupo).

† Comparación intra sexos (entre masculino y masculino, femenino y femenino de ambos grupos).

Tabla 3. Comparación de ancho maxilar entre pacientes con secuela de FLAPU y pacientes no fisurados de 6 a 15 años atendidos en el IESN 2000-2009.

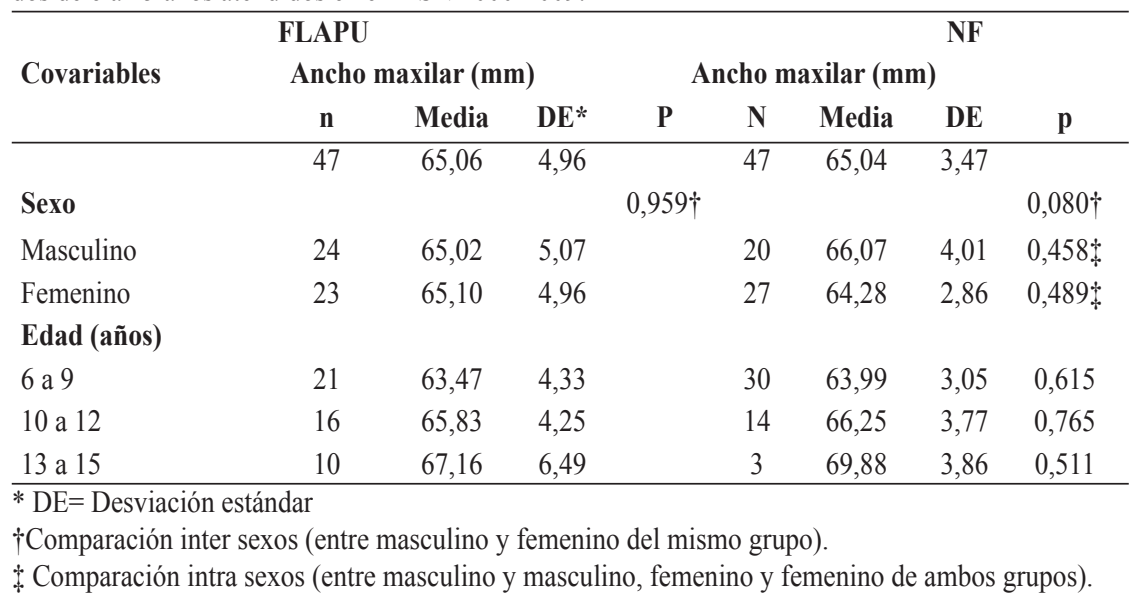

por el incremento en el ancho de varias partes faciales (8); sin embargo, esta diferencia no fue significativa. Esto puede deberse a que

la cantidad de tejido palatino al momento de la queiloplastia influye en el crecimiento transversal maxilofacial, variable que no se pudo con- 
trolar en el presente estudio.

Las diferencias entre los anchos esqueléticos de pacientes con secuela de FLAPU y NF pueden estar enmascaradas por las características morfofaciales clínicas de los pacientes, ya que según Ramos et al. (17), los individuos braqui, meso y dolicofaciales pueden pertenecer a una clasificación morfofacial distinta a nivel esqueletal.

En el grupo de NF al comparar las dimensiones transversales esqueléticas (ancho maxilar y ancho facial) entre ambos sexos se encontró una diferencia significativa para el ancho facial siendo mayor en el sexo masculino y menor en el femenino, lo cual concuerda con Lux et al. (18) que encontró en una población de niños no fisurados de 7 a 15 años que la mayoría de anchos craneofaciales fueron mayores en hombres que en mujeres. Echaniz (19) encontró algo similar, cuando los sujetos de su muestra poseían dentición mixta, todos los valores craneofaciales eran mayores en varones que en mujeres y estadísticamente muy significativos. Podría suponerse que esta diferencia entre dimensiones transversales esqueléticas entre hombres y mujeres estaría relacionada a una herencia genética ligada al cromosoma $\mathrm{Y}$, ya que siempre se encuentran mayores en el sexo masculino, hecho que debe estudiarse más a fondo.

En las dimensiones transversales del arco maxilar (ancho intercanino y ancho intermolar) se encontraron diferencias altamente significativas en el ancho intercanino, siendo menores en los pacientes con secuela de FLAPU al compararlos con los pacientes NF en los grupos de 6 a 9 y de 10 a 12 años. Resultados similares a los obtenidos por Dibiase et al. (20) y Da Silva Filho

Tabla 4. Comparación de ancho facial entre pacientes con secuela de FLAPU y pacientes no fisurados de 6 a 15 años atendidos en el IESN 2000-2009.

\begin{tabular}{lcccccccc}
\hline & \multicolumn{3}{c}{ FLAPU } & \multicolumn{5}{c}{ NF } \\
Covariables & Ancho facial (mm) & \multicolumn{5}{c}{ Ancho facial (mm) } \\
& $\mathbf{n}$ & Media & $\mathbf{D E}^{*}$ & $\mathbf{P}$ & $\mathbf{N}$ & Media & $\mathbf{D E}$ & $\mathbf{p}$ \\
\hline & 47 & 125,50 & 5,42 & & 47 & 124,38 & 6,60 & \\
Sexo & & & & $0,732 \dagger$ & & & & $0,040 \dagger$ \\
Masculino & 24 & 125,23 & 5,18 & & 20 & 126,66 & 7,41 & $0,475 \dagger$ \\
Femenino & 23 & 125,78 & 5,77 & & 27 & 122,70 & 5,48 & $0,055 \ddagger$ \\
Edad (años) & & & & & & & & \\
6 a 9 & 21 & 123,19 & 4,77 & & 30 & 122,30 & 4,53 & 0,467 \\
10 a 12 & 16 & 126,42 & 5,52 & & 14 & 126,09 & 6,52 & 0,886 \\
13 a 15 & 10 & 128,91 & 4,70 & & 3 & 137,18 & 9,80 & 0,059 \\
\hline
\end{tabular}

* DE= Desviación estándar

$\uparrow$ Comparación inter sexos (entre masculino y femenino del mismo grupo).

$\$$ Comparación intra sexos (entre masculino y masculino, femenino y femenino de ambos grupos).

et al. (9) en poblaciones europeas y brasileras respectivamente. Diferencia que se puede explicar debido a que la falta de estructura ósea sumada a la respuesta biológica del crecimiento transversal que acontece luego de la cirugía en la zona anterior del maxilar se encuentra restringida por el tejido cicatrizal que se forma. Para el grupo de 13 a 15 años no se encontró diferencia estadísticamente significativa. Ya que en este grupo etario, con frecuencia se realizan injertos óseos para corregir defectos encontrados y poder así devolver en cierta cantidad la conformidad del paladar y del alveolo, junto con ello mejorar posición de los caninos. Para el ancho intermolar no se encontraron diferencias estadísticamente significativas entre ambos grupos de pacientes en los tres grupos de edad. Esto se podría explicar ya que normalmente las fisuras que involucran la zona posterior del paladar no son muy frecuentes, hecho que conduce a que no existe diferencia marcada de esta dimensión.

En ambos grupos, en los pacientes con secuela de FLAPU y los NF al comparar las dimensiones transversales del arco maxilar (ancho intercanino y ancho intermolar) entre ambos sexos, no se encontra- ron diferencias estadísticamente significativas, lo cual difiere de los resultados obtenidos por Dibiase et al. (20), Da Silva Filho et al. (9), y Echaniz (19), que encontraron las dimensiones mayores en el sexo masculino, lo que nos indicaría que no existe en esta dimensiones un factor hereditario ligado al sexo.

Las conclusiones del presente estudio son:

1. En relación a las dimensiones transversales del arco maxilar, el ancho intercanino fue menor en los grupos de 6 a 9 años y de 10 a 12 años de edad en pacientes con secuela de FLAPU al compararlos con pacientes no fisurados.

2. No existen diferencias entre las dimensiones transversales esqueléticas (ancho maxilar y ancho facial) de pacientes con secuela de FLAPU y pacientes no fisurados, en los tres grupos etarios estudiados

Agradecimientos

A los doctores de la Facultad de Odontología de la UNMSM: Héctor Marengo, Leoncio Menéndez, Fernando Pérez, Ana María Díaz, y a los doctores del IESN: Lourdes 
Motta, Gina Bustamante, Cesar Villaverde, Alejandro Cornejo, Olinda Huapaya, Luis Rodríguez por su incondicional apoyo, asesoría y consejos en la realización del presente trabajo de investigación.

\section{Referencias Bibliográficas}

1. Mogollón L. Prevalencia de agenesia dentaria y dientes supernumerarios en pacientes con fisura labio alveolo palatina atendidos en el Instituto Especializado de Salud del Niño entre los años 2005 -2008. Tesis Bachiller. Lima, Perú: Universidad Nacional Mayor de San Marcos, 2008.

2. Caro M, Quevedo C, Pereira Y. Descripción de las dimensiones de arco maxilar de pacientes con dentición permanente que presentan secuela de labio y paladar fisurado unilateral completo antes y durante el tratamiento ortodóntico. Rev Cient Bogotá. 2002; 8(1): 47-59.

3. Román N. Guía Clínica: Maloclusión por secuela de fisura labio palatina. Lima: Unidad de Fisurados Instituto Especializado en Salud del Niño; 2008.

4. Da Silva OG, Ramos AL, Camargo RC. The influence of unilateral cleft lip and palate on maxillary dental arch morphology. Angle Orthod. 1992;62: 283-90.

5. Sunjay S, Ashok U, Niranjan K, Mago S. Craniofacial computerized tomography analysis of the midface of patients with repaired complete unilateral cleft lip and palate. Am J Orthod Dentofacial Orthop. 2008;134(3):41829.

6. Honda Y, Suzuki A, Nakamura N, Ohishi M. Relationship between primary palatal form and maxillofacial growth in japane- se children with unilateral cleft lip and palate: Infancy to Adolescence. Cleft Palate-Cran J. 2002; 39(5): 527-534.

7. Marcusson A, Paulin G. Changes in occlusion and maxillary dental arch dimensions in adults with treated unilateral complete cleft lip and palate: a follow-up study. Eur J Orthod. 2004;26(4):385-390.

8. Motohashi N, Kuroda T, Capelozza Filho L, de Souza Freitas. P-A Cephalometric analysis of nonoperated adult cleft lip and palate. Cleft Palate - Cran J. 1994; 31(3): 193-200.

9. Da Silva Filho O, Monteiro de Castro F, Coelho de Andrade A, de Souza Freitas J, Bishara S. Upper dental arch morphology of adult unoperated complete bilateral cleft lip and palate. Am J Orthod Dentofacial Orthop. 1998;114:154-61.

10.Linkevièienë L, Olekas J, Zaleckas L, Kapuðinskas G. Relation between the severity of palatal cleft and maxillary dental arch size. Act Med Lituanica. 2005;12(1): 54-57.

11. Molsted K, Dahl E. Asymmetry of the maxilla in children with complete unilateral cleft lip and palate. Cleft Palate-Cran J. 1990;27(2): 184-192.

12.Da Silva F, Correa N, Capelozza F. Mandibular growth in patients with cleft lip and/or cleft palate-the influence of the type. Am J Orthod Dentofacial Orthop 1993;104(3): 269-275

13. Moyers RE, Van der Linden F, Riolo MI, McNamara JA. Standard for human occlusal development. Monograph 5. Craniofacial Growth Series. Center For Human Growth and development. An Arbor (US):: University of Michigan; 1976.
14. Ricketts RM. Perspectives in the clinical application of cephalometrics. The first fifty years. Angle Ortho. 1981;51(2):115-50

15. Gregoret J. Cefalometría frontal. En: Gregoret J, Tuber E, Escobar LH, Matos A. (Editores). Ortodoncia y cirugía ortognática, diagnóstico y planificación del tratamiento. 2da Ed. Barcelona:Editorial Publicaciones Médicas;1997:p.211-224.

16. Romani N. Correlación entre el ancho transpalatino con el ancho maxilar y facial en escolares de 8 a 10 años de edad. Tesis Bachiller. Lima, Perú: Universidad Nacional Mayor de San Marcos, 2003.

17.Ramos P, Suazo G, Martinez, M, Reyes L. Relaciones transversales faciales en niños chilenos de la Región del Maule. Int J Morphol. 2007; 25(4):703707.

18.Lux C, Conradt C, Burden D, Komposch G. Transverse development of the craniofacial skeleton and dentition between 7 and 15 years of age-a longitudinal postero-anterior cephalometric study. Eur J Orthod. 2004; 26(1):31-42.

19.Echaniz R. Comprobación de las medidas transversales de las arcadas y del índice de Izard. Tesis Doctoral. Madrid, España: Universidad Complutense de Madrid, 1994.

20.DiBiase A, DiBiase D, Hay N, Sommerlad B. The relationship between arch dimensions and the 5-year index in the primary dentition of patients with complete UCLP. Cleft Palate - Cran J. 2002; 39(6): 635-640. 